\title{
ALMOST EVERY ORLICZ SPACE IS ISOMORPHIC TO A STRICTLY CONVEX ORLICZ SPACE
}

\author{
M. M. RAO ${ }^{1}$
}

1. Introduction. The purpose of this note is to prove that every Orlicz space associated with a continuous Young's function is isomorphic to another strictly convex Orlicz space, and to add some related remarks. Let $\Phi$ be a nontrivial symmetric convex function vanishing at the origin. Then $\Phi$ is termed a Young's function if either $\Phi$ is discontinuous, in which case $\Phi(x)<\infty$ for $|x|<x_{0}<\infty$ and $=\infty$ for $|x|>x_{0}$, or $\Phi$ is continuous and $\Phi(x)>0$ for $x>0$. It is termed a generalized Young's function otherwise so that when it is continuous $\Phi(x)=0$ and $0 \leqq x \leqq a, a>0$, is also possible. These are, except for the classification, the same definitions as given in [4] or [7]. If $\Phi$ is a generalized Young's function let $L^{\Phi}$ be the Orlicz space on a nontrivial measure space $(\Omega, \Sigma, \mu)$ (i.e., every set of positive $\mu$-measure has a subset of positive finite $\mu$-measure).

As remarked in $[4$, p. 93, equation (10.13)] and in the constructions in [4, pp. 20-21], every continuous Young's function $\Phi$ is equivalent to a strictly convex Young's function $\Phi_{1}$ whose derivative $\Phi_{1}^{\prime}$ (which exists a.e. (Lebesgue)) is also continuous. In fact, simply take $\Phi_{1}^{\prime}(u)=\Phi^{\prime}(u)(1+(u / n))$ for $n-1 \leqq u \leqq n, n \geqq 1$, so that it is continuous, and let $\Phi_{1}$ be the indefinite integral of $\Phi_{1}^{\prime}$ and define $\Phi_{1}(-x)$ $=\Phi_{1}(x)$. Then $\Phi_{1}$ is a strictly convex Young's function and $\Phi(u)$ $\leqq \Phi_{1}(u) \leqq 2 \Phi(u)$ for all $u$ so that they are equivalent (in the sense of $[4$, p. 15]). In this note the following two results will be proved. (Note that here $\Phi^{\prime}$ may be (and is) assumed continuous, as in $[8$, p. 25], by adjoining to the curve $y=\Phi^{\prime}(x)$ the vertical segments at the countable set of discontinuities. Thus, with this normalization, $\Phi^{\prime}$ will be continuous if $\Phi$ is. Also isomorphism stands for topological isomorphism.)

Theorem 1. If $\Phi$ is a continuous Young's function and $L^{\Phi}$ is the associated Orlicz space on an arbitrary (nontrivial) measure space $(\Omega, \Sigma, \mu)$, then $L^{\Phi}$ is isomorphic to a strictly convex Orlicz space.

If $\mu$ is a finite measure, a completely general statement holds.

THEOREM 2. If $\Phi$ is any generalized Young's function and $L^{\Phi}$ is the associated Orlicz space on a finite measure space $(\Omega, \Sigma, \mu)$, then $L^{\Phi}$ is isomorphic to a strictly convex Orlicz space.

Received by the editors January 12, 1967.

1 Supported under the NSF Grant GP-5921. 
These results contain the main result in [6] as a special case. In fact in [6] it was assumed that $\Phi(2 x) \leqq C \Phi(x)$ for $0<C<\infty$, the $\Delta_{2}$-condition of [4]. Using the construction given in [4, pp. 28-29], one can easily construct between any given pair of Lebesgue spaces an Orlicz space $L^{\Phi}$ such that $\Phi$ does not satisfy the above growth condition so that, in a certain well-defined sense, the Young's functions satisfying the growth condition form a small class among all continuous Young's functions. Proofs of the above results will be given in the next section and some comments about certain related results will be given in the last section.

\section{Proofs.}

Proof of Theorem 1. Since $\Phi$ is a continuous Young's function, by the remark above Theorem 1 , there exists a $\Phi_{1}$ which is strictly convex with $\Phi_{1}^{\prime}$ continuous and such that $\Phi(x) \leqq \Phi_{1}(x) \leqq 2 \Phi(x)$ for all $x$. From this, it follows that $L^{\Phi}$ and $L^{\Phi_{1}}$ have the same elements and have equivalent norms (an immediate consequence of the definition of norms, cf. [4], [5] or [7]-there are two equivalent norms in these spaces, cf. $[4$, p. 80$]$, and any one can be considered). But $L^{\Phi_{1}}$ is strictly convex (= rotund) by [5, Theorem 4, p. 681] since $\Phi_{1}$ is strictly convex and $\Phi_{1}^{\prime}$ is continuous. Hence $L^{\Phi}$ and $L^{\Phi_{1}}$ are (topologically) isomorphic, and the proof is complete.

Proof of Theorem 2. First let $\Phi$ be a continuous generalized Young's function. Then there exists a continuous (ordinary) Young's function $\Phi_{1}$ such that $\Phi(x)=\Phi_{1}(x)$ for $|x| \geqq x_{1}>0$ for some $x_{1}$. This follows from [4, p. 17], where $\Phi$ is called a principal part of $\Phi_{1}$. In fact there is an $x_{0}>0$ such that $\Phi(x)>0$ for $|x|>x_{0}$. Choose $x_{1} \geqq x_{0}+1$ so that $x_{1} \Phi^{\prime}\left(x_{1}\right) \geqq x_{0} \Phi^{\prime}\left(x_{1}\right)+\Phi\left(x_{0}\right)>0$. This is possible since $\Phi(x) \rightarrow \infty$ as $x \rightarrow \infty$. Define $\alpha=\left[x_{1} \Phi^{\prime}\left(x_{1}\right)\right]\left[\Phi\left(x_{1}\right)\right]^{-1}$ which is greater than unity, and set

$$
\begin{aligned}
\Phi_{1}(x) & =\left[\Phi\left(x_{1}\right) / x_{1}^{\alpha}\right]|x|^{\alpha}, & & |x| \leqq x_{1}, \\
& =\Phi(x), & & |x| \geqq x_{1} .
\end{aligned}
$$

Then $\Phi_{1}$ is a continuous Young's function with $\Phi$ as its principal part. Since $\mu(\Omega)<\infty$, it follows from $\left[4\right.$, p. 112] that $L^{\Phi}$ and $L^{\Phi_{1}}$ have the same elements and their norms are equivalent. But by Theorem 1 above, $L^{\Phi_{1}}$ is isomorphic to a strictly convex Orlicz space and hence $L^{\Phi}$ has the same property, and the result follows in this case.

If $\Phi$ is discontinuous then $L^{\Phi} C L^{\infty}$ in general (cf. [7, p. 82]) and $L^{\Phi}=L^{\infty}$ if $\mu(\Omega)<\infty$. Moreover it is easily seen that $\|f\|_{\infty} \leqq k\|f\|_{\Phi}$, which is the reason for the above inclusion $\left(\|\cdot\|_{\infty}\right.$ is $L^{\infty}$-norm and $\|\cdot\|_{\Phi}$ is $L^{\Phi}$-norm); and it follows by the closed graph theorem and the 
fact $L^{\Phi}=L^{\infty}$ that the two norms are actually equivalent, so that these spaces are isomorphic. But, by [1, Theorem 11], $L^{\infty}$ is isomorphic to a strictly convex space (a subspace of $\left(L^{1}\right)^{*}$, the adjoint of $L^{1}$ ). These two statements imply the truth of the theorem in this case, and the proof is complete, since now $\left(L^{1}\right)^{*}$ is an Orlicz space (cf. e.g., [5, Theorem 1]; or [7]).

\section{Remarks.}

1. It is a consequence of Theorems 1 and 2 above and immediate from [5, Theorem 4] that every reflexive Orlicz space, on an arbitrary measure space, is isomorphic to a strictly convex (and smooth) Orlicz space. The hypothesis of [5, Theorem 4$]$ is necessary and sufficient for reflexivity and is a consequence of a theorem of Halperin [3, p. 205].

2. It is interesting to note that the $L^{1}$-space is isomorphic to a strictly convex Orlicz space $L^{\Phi_{1}}$ and not to a Lebesgue space, where $\Phi_{1}(x)=|x|+\left(x^{2} / 2 n\right), n-1 \leqq|x| \leqq n$, as seen in the proof above of Theorem 1 where $\Phi(x)=|x|$ for the $L^{1}$ space. This will give an appreciation of the proof of [1]. The strictly convex $\Phi_{1}$ associated with the $\Phi$ is, of course, not unique.

3. The (negative) results of [2] can be used to show that the hypotheses of Theorems 1 and 2 cannot be materially improved.

4. It may be noted that the results of [6] are actually contained in the earlier results [5, Part II] of which the above two theorems are extensions. The result of Theorem 1 was briefly indicated [5, top p. 674]. Perhaps it was too condensed to recognize the significance, and the elaboration given here should therefore be of interest.

\section{REFERENCES}

1. M. M. Day, Strict convexity and smoothness of normed spaces, Trans. Amer. Math. Soc. 78 (1955), 516-528.

2. - Every L-space is isomorphic to a strictly convex space, Proc. Amer. Math. Soc. 8 (1957), 415-417. 208.

3. I. Halperin, Reflexivity in the $L^{\lambda}$-function spaces, Duke Math. J. 21 (1954), 205-

4. M. A. Krasnosel'skiY and Ya. B. Ruticki1, Convex functions and Orlicz spaces, translated from Russian, Noordhoff, Groningen, 1961.

5. M. M. Rao, Smoothness of Orlicz spaces, Indag. Math. 27 (1965), 671-690.

6. K. Sundaresan, Orlicz spaces isomorphic to strictly convex spaces, Proc. Amer. Math. Soc. 17 (1966), 1353-1356.

7. A. C. Zaanen, Linear analysis, North-Holland, Amsterdam, 1953.

8. A. Zygmund, Trigonometric series, Vol. I, 2nd ed., Cambridge Univ. Press, New York, 1959.

Carnegie Institute of Technology 